\title{
Raman microscopy of bladder cancer cells expressing green fluorescent protein
}

Gurjit S. Mandair

Amy L. Han

Evan T. Keller

Michael D. Morris 


\title{
Raman microscopy of bladder cancer cells expressing green fluorescent protein
}

\author{
Gurjit S. Mandair, ${ }^{a, *}$ Amy L. Han, ${ }^{b}$ Evan T. Keller, ${ }^{b}$ and Michael D. Morris ${ }^{c}$ \\ aUniversity of Michigan, School of Dentistry, Department of Biologic and Materials Sciences, 1011 North University Avenue, Ann Arbor, Michigan \\ 48109-1078, United States \\ bUniversity of Michigan, Department of Urology and Biointerfaces Institute, NCRC Building 20, 2800 Plymouth Road, Ann Arbor, Michigan \\ 48109-2800, United States \\ 'University of Michigan, Department of Chemistry, 930 North University Avenue, Ann Arbor, Michigan 48109-1055, United States
}

\begin{abstract}
Gene engineering is a commonly used tool in cellular biology to determine changes in function or expression of downstream targets. However, the impact of genetic modulation on biochemical effects is less frequently evaluated. The aim of this study is to use Raman microscopy to assess the biochemical effects of gene silencing on T24 and UMUC-13 bladder cancer cell lines. Cellular biochemical information related to nucleic acid and lipogenic components was obtained from deconvolved Raman spectra. We show that the green fluorescence protein (GFP), the chromophore that served as a fluorescent reporter for gene silencing, could also be detected by Raman microscopy. Only the gene-silenced UMUC-13 cell lines exhibited low-to-moderate GFP fluorescence as determined by fluorescence imaging and Raman spectroscopic studies. Moreover, we show that gene silencing and cell phenotype had a greater effect on nucleic acid and lipogenic components with minimal interference from GFP expression. Gene silencing was also found to perturb cellular protein secondary structure in which the amount of disorderd protein increased at the expense of more ordered protein. Overall, our study identified the spectral signature for cellular GFP expression and elucidated the effects of gene silencing on cancer cell biochemistry and protein secondary structure. ๑ 2016 Society of Photo-Optical Instrumentation Engineers (SPIE) [DOI: 10.1117/1.JBO.21.11.115001]
\end{abstract}

Keywords: Raman microscopy; bladder cancer; green fluorescent protein; gene silencing; protein secondary structure.

Paper 160392R received Jun. 10, 2016; accepted for publication Oct. 14, 2016; published online Nov. 2, 2016.

\section{Introduction}

Green fluorescent protein (GFP) is widely used in fundamental and applied research. In cell biology, GFP is used to monitor gene expression and localize proteins in living cells, including the ability to track a particular cell type within a given heterogeneous population of cells, tissues, or organ systems. ${ }^{1}$ The ability to document the proliferation, migration, and invasion of particular cell types is especially critical for understanding the pathogenesis of cancer metastasis. For example, GFP expression in cancer cells allows them to be tracked in blood and lymphatic vessels and metastatic sites, including bone in vivo. ${ }^{2}$

Structurally, GFP is a folded protein with a unique $11 \beta$-sheet barrel-like scaffold tethered to an $\alpha$-helical segment that runs through its center. ${ }^{3,4}$ The critical light sensitive chromophore of GFP is the Ser-Try-Gly tripeptide sequence located near the center of the folded protein. The posttranslational cyclization of this tripeptide leads to the formation of the fluorescent 4( $p$-hydroxybenylidene)-imidazolidin-5-one chromophore. In addition to characterization by multiple biophysical techniques, ${ }^{5}$ the GFP chromophore and its structural variants have also been studied by Raman spectroscopy. In such studies, Raman spectroscopy has provided detailed chemical and structural information on the chromophore's microenvironment in response to changes in $\mathrm{pH}$ and excitation state. ${ }^{6,7}$ However, the spectroscopic signature of the GFP chromophore has yet to be detected from stable cells transduced with the GFP reporter gene.

*Address all correspondence to: Gurjit S. Mandair, E-mail: gmandair@umich .edu
While stable GFP-transduced cells can be obtained, their biochemical and protein structural properties need to be evaluated to ensure that they are consistent with the cell type under study. ${ }^{8}$ A recent proteomic study has shown that GFP expression in breast cancer cells could alter the abundance of a number of proteins associated with protein folding. ${ }^{9}$ In this study, we propose that Raman microscopy can not only be used to differentiate between different cancer cell lines generated by small hairpin RNA (shRNA)-mediated gene silencing, but could also be used to assess GFP-induced biochemical and/or protein structural changes. This study compares subsets of gene-silenced cell lines obtained from two human bladder cancer cell lines (T24 and UMUC-13) transduced with the GFP reporter gene.

\section{Materials and Methods}

\subsection{Cell Cultures}

T24 and UMUC-13 (UC13) bladder cancer cell lines were provided by Dr. Monica Liebert (University of Michigan, Ann Arbor). Parental T24 cells originated from human urinary bladder carcinoma, while UMUC-13 cells were derived from lymphatic metastases of the transitional cell carcinomas of the bladder. ${ }^{10,11}$ All cells were maintained in Dulbecco's modified Eagle medium, high glucose [Gibco-Life Technologies (Gibco); Carlsbad, California] supplemented with $10 \%$ fetal bovine serum, $100 \mathrm{U} / \mathrm{ml}$ penicillin, and $100 \mu \mathrm{g} / \mathrm{ml}$ streptomycin in a humidified atmosphere of $5 \% \mathrm{CO}_{2}$ at $37^{\circ} \mathrm{C}$. Cells were

$1083-3668 / 2016 / \$ 25.00$ (c) 2016 SPIE 
passaged with $0.05 \%$ trypsin-ethylenediamine tetra-acetic acid (Gibco) when confluent (2 to 3 days). Both parental T24 (T24Con) and UC13 (UC13-Con) cells were transduced with different lentiviral particles containing a GFP reporter. The lentiviral particles also contained two different constructs, shRNA A and shRNA C, which were transduced separately into both T24-Con and UC13-Con cell lines to generate the different respective knockdown cell lines: T24-A, T24-C, UC13-A, and UC13-C. Scrambled T24-Scr and UC13-Scr cell lines were also generated to rule out nonspecific target effects of the shRNA plasmid and to provide negative controls for the gene specific knockdown. All cell lines were grown on Petri dishes $(n=5$ per cell line), including two eight-well chamber LabTek II slides (Nunc, Rochester, New York) for DNA staining and fluorescence imaging.

Cell viability was determined by the trypan blue exclusion test. Briefly, $10 \mu \mathrm{L}$ of the cell suspension was mixed with an equal volume of $0.4 \%$ trypan blue stain (Gibco; Carlsbad, California) in a microwell plate. The $10-\mu \mathrm{L}$ aliquots of the cell suspensions were loaded into the dual-chambered counting slide and the cells were counted using the TC20 Automated Cell Counter (Bio-Rad Laboratories). Percent cell viability was calculated using the following equation: cell viability $=100-$ $(100 /$ total number of cells $\times$ number of dead or damaged cells). High cell viability values of $93 \%$ to $100 \%$ were routinely obtained for all cell lines used in this study.

\subsection{Cell Sample Preparation}

Trypsinized cell pellets were resuspended in $5 \mathrm{~mL}$ phosphate buffered saline (PBS), centrifuged at low speed (1000 rpm for 3 mins), and then the supernatant was discarded. Cell pellets were further washed twice with $1 \mathrm{~mL}$ PBS to ensure all traces of spent culture media and trypsin were removed. Recovered cell pellets were diluted at a ratio of 1:3 with PBS in order to produce a suitable cell density for Raman microscopy. Aliquots of the diluted cell suspensions ( $1 \mu \mathrm{L}$ per cell line) were then deposited onto the gold-coated microscope slide (EMF Corporation, Ithaca, New York). The deposits were desiccated over silica beads using the procedure described elsewhere. ${ }^{12}$ The desiccated circular deposits $(\sim 2.72 \pm 0.07 \mathrm{~mm}$ in diameter $)$ appeared granular under a light microscope because of the rounded shape of the detached cells. ${ }^{13}$

\subsection{Staining and Fluorescence Imaging}

Culture media were removed from confluent cell lines cultured on the chamber slides ( $n=2$ per cell line). The cells were immediately fixed with $0.2 \mathrm{~mL}$ ice-cold methanol $(5 \mathrm{mins})$ and then rinsed twice with $0.4 \mathrm{~mL}$ ice-cold PBS (2 mins then $1 \mathrm{~min}$ ). After removing PBS, the cells were incubated with $0.1 \mathrm{~mL}$ of $0.1 \mu \mathrm{g} / \mathrm{mL}$ 4',6-diamidino-2-phenylindole (DAPI, SigmaAldrich, St. Louis, Missouri) solution (3 mins). After DNA staining with DAPI, the cells were rinsed three times with $0.4 \mathrm{~mL}$ PBS $(3 \times 5$ mins $)$. The medium chamber was removed and the slide was mounted by a coverslip with a drop of mounting medium. The coverslip was sealed with nail varnish to prevent the cells from drying. The cells were examined using an Olympus BX41 microscope, equipped with an Olympus PP73 camera and CellSens image-processing software. An Olympus UHGLGPS light guide-coupled illumination system served as the fluorescence light source for blue (DAPI) and green (GFP) fluorescence imaging. Images were acquired using a $40 \times 0.75 \mathrm{NA}$ objective and fixed acquisition times of $600 \mu \mathrm{s}$ and $500 \mathrm{~ms}$, respectively. Images were analyzed using the CellSens imaging-processing software. Areas of the images were estimated using a Formvar-coated copper grid (100 mesh; Electron Microscopy Sciences, Hatfield, Pennsylvania).

\subsection{Raman Microscopy and Spectral Analysis}

The 785-nm Raman microprobe was constructed locally and is described in detail elsewhere. ${ }^{14,15}$ The excitation laser was shaped into a line and focused through a $20 \times / 0.75$ NA objective with a laser power output of $\sim 30 \mathrm{~mW}$ at the sample. The laser line was $\sim 135 \mu \mathrm{m}$ in length with a lateral resolution of $\sim 15 \mu \mathrm{m}$ (laser area $\sim 2025 \mu \mathrm{m}^{2}$ ). This Raman line-scan approach enabled a large number of cells to be analyzed simultaneously compared to single cell collection approaches. The number of cells irradiated by the laser line (cells $/ \mathrm{mm}^{2}$ laser area) is also estimated from the light microscope images. Spectra were collected at 6 to 8 different locations from 2 to 3 dried cellular deposits ( $n=5$ per cell line). The cosmic ray removal setting was used prior to collecting the spectra using an accumulation cycle time of 7 mins $(7 \times 60 \mathrm{~s})$.

All Raman data were processed in MATLAB ${ }^{\circledR}$ software using locally written scripts described elsewhere. ${ }^{16}$ The script also included an automated "adaptive min-max" polynomial fitting procedure (third order, constrained) to correct for background fluorescence. ${ }^{17}$ All spectra were imported into GRAMS/ $\mathrm{AI}^{\circledR}$ software for baseline correction and normalization against the proteinllipid $\mathrm{CH}_{2}$ deformation band at $\sim 1447 \mathrm{~cm}^{-1}$. For curve-fitting, standard second derivative and constrained Gaussian deconvolution functions ${ }^{18}$ were applied to the following spectral ranges: 685 to $840 \mathrm{~cm}^{-1}(698,718,744,757,781$, 810 , and $826 \mathrm{~cm}^{-1}$ ), 1180 to $1400 \mathrm{~cm}^{-1}$ (amide III region: $1206,1229,1248,1267,1280,1297,1316,1337$, and $1356 \mathrm{~cm}^{-1}$ ), and 1530 to $1720 \mathrm{~cm}^{-1}$ (amide I region: 1550,1570 , $1584,1605,1619,1635,1656,1672,1685$, and $1698 \mathrm{~cm}^{-1}$ ). All band intensities were nonnegative and were recorded when the best-fit curves yielded $r^{2}$ values of 0.98 or more.

Raman band assignments pertinent to cancer cells and the GFP chromophore were identified and their relative intensities measured. Only ratiometric Raman parameters derived from select band intensities that yielded the most significant results will be presented here. For GFP expression and biochemical changes, the following ratiometric parameters were calculated: GFP/amide (Am) I $\alpha$-helix $\left(1550 / 1656 \mathrm{~cm}^{-1}\right)$, nucleic acid/ tryptophan (Typ) $\left(781 / 757 \mathrm{~cm}^{-1}\right)$, Typ/phospholipid (757/ $\left.718 \mathrm{~cm}^{-1}\right)$, and phospholipid/cholesterol $\left(718 / 700 \mathrm{~cm}^{-1}\right)$ ratios. ${ }^{19-21}$ For changes in protein secondary structure, two ratiometric parameters were calculated: Am III disorder/ order $\left(1248 / 1266 \mathrm{~cm}^{-1}\right)$ and Am I disorder $/ \alpha$-helix (1685/ $\left.1656 \mathrm{~cm}^{-1}\right) .^{22,23}$ Data were presented as mean \pm standard deviation (SD). Significant differences between parental and gene-silenced cell lines, including scrambled controls, were analyzed using one-way analysis of variance with statistical significance defined at $p<0.05$.

\section{Results and Discussion}

\subsection{Spectral Analysis of Dried Cellular Deposits}

The mean Raman spectra of different parental and gene-silenced T24 and UC13 cell lines deposited on gold-coated substrates are shown in Fig. 1. All spectra exhibited similar compositional and 


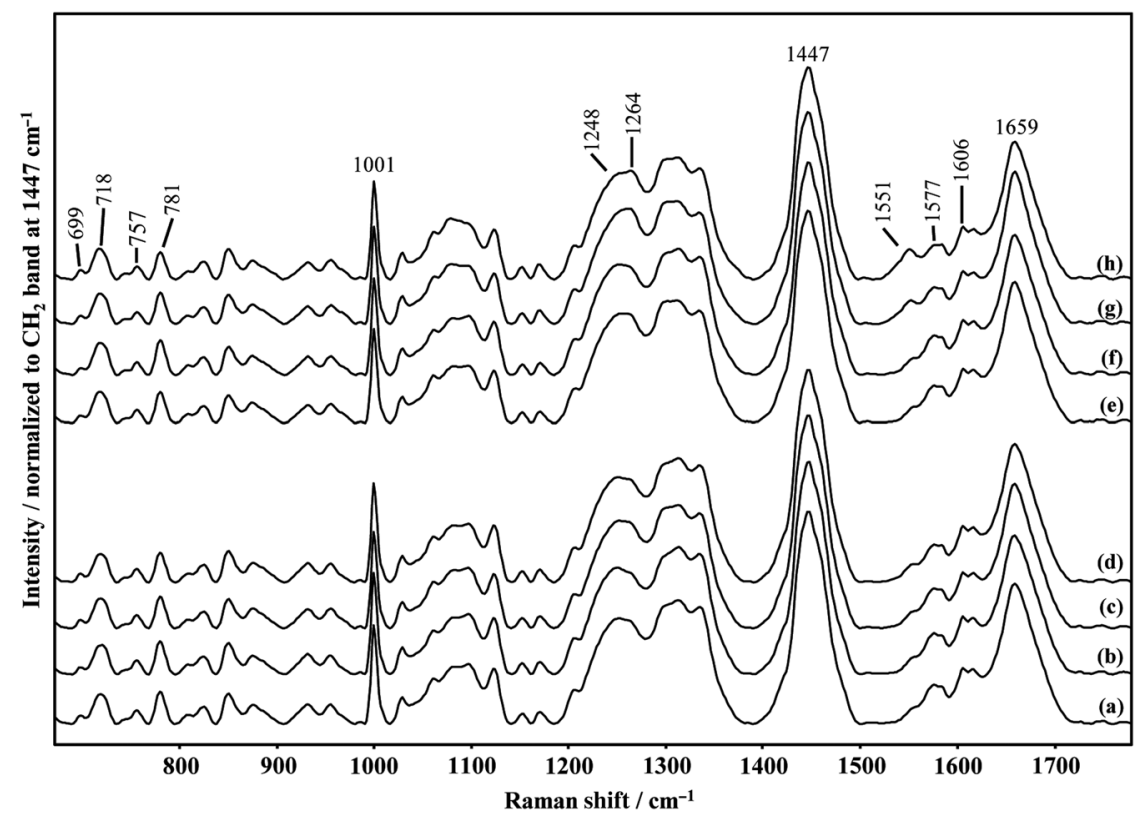

Fig. 1 Representative baselined-corrected Raman spectra (averaged from multiple locations) for the following T24 and UC13 bladder cancer cells desiccated on gold-coated substrates: (a) T24-Con, (b) T24-Scr, (c) T24-A, (d) T24-C, (e) UC13-Con, (f) UC13-Scr, (g) UC13-A, and (h) UC13-C.

structural profiles but with subtle differences that will be discussed in more detail later. The consistent quality of the spectra was attributed to several factors. First of all, the high percent cell viability levels (93\% or more) obtained at each cell passage ensured that the cell populations used in the Raman spectroscopic analysis were free from dead or damaged cells. ${ }^{24}$ Second, by using the laser-line collection mode, Raman scatter from a larger collection of individual cells could be obtained, which could be further enhanced if the cells were preconcentrated by centrifugation prior to deposition and analysis. Indeed, our image analyses show that the later approach achieved $\sim 13$ cells $/ \mu \mathrm{m}^{2}$ laser areas compared to $\sim 3$ cells $/ \mu \mathrm{m}^{2}$ laser areas, if cells grown on a slide had been analyzed. Figure 2 shows the granular appearance of desiccated UC13-Scr cells deposited on gold-coated substrates. The rounded morphology exhibited by UC13-Scr cells was typical for all cell lines used in

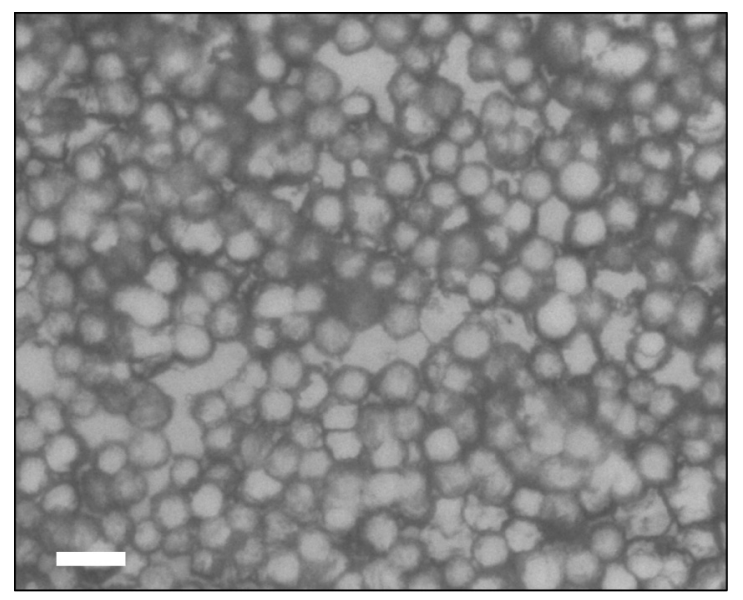

Fig. 2 Light microscope image showing the granular appearance of detached UC13-Scr cells desiccated on gold-coated substrate. Scale bar: $20 \mu \mathrm{m}$. this study and was similar in appearance to those reported for breast carcinoma and myeloid leukemia cells dried on $\mathrm{CaF}_{2}$ substrates. ${ }^{13}$ Third, cells deposited on gold-coated substrates exhibited good spectral signal quality that could be attributed to the substrate's low background signal contribution and reflective properties when compared to transparent quartz substrates. ${ }^{25,26}$

The use of desiccation to fix cells has been shown to increase spectral reproducibility and signal-to-noise ratios. ${ }^{12}$ Another study showed that spectra from dry-fixed and live cells were highly correlated, implying that dried-fix cells could be used as proxies for living cells. ${ }^{27}$ Providing that one fixation method is consistently maintained throughout the study, fixationinduced spectral effects can be minimized, thereby permitting useful comparisons between different cell types and disease states. ${ }^{28}$ For instance, Raman microscopy in conjunction with linear discriminate analyses successfully differentiated between erythrocyte, leukocyte, acute myeloid leukemia, and breast tumor cells trapped inside microfluidic devices with high accuracies. $^{29}$ Moreover, the study showed that accuracies were comparable to previous studies in which the same cell types were air-dried or fixed on Petri dishes. In this current study, desiccation was chosen over solvent and chemical fixation methods in order to minimize the potential loss of cell membrane lipid components, modifications to protein secondary structure, and/or changes to nucleolar protein concentrations. ${ }^{12,30}$ Indeed, a recent spectroscopic study with principal component analysis showed that membrane lipid was a key differentiating character trait between different cancer cell lines. ${ }^{31}$

The Raman spectra of cells are complex because the microscopy technique provides simultaneous information on intracellular proteins, amino acids, nucleic acids, lipids, and cholesterol molecules. The major protein-related and nucleic acid bands included the Typ band at $\sim 757 \mathrm{~cm}^{-1}$ and the DNA/RNA band at $\sim 781 \mathrm{~cm}^{-1}$, respectively. ${ }^{20}$ Bands at $\sim 700$ and $\sim 718 \mathrm{~cm}^{-1}$ were assigned to cholesterol (mixed contributions from 
cholesterol esters) and phospholipid (mainly phosphocholine) vibrations, respectively. ${ }^{21,32}$ For protein secondary structures, tentative band assignments were proposed for the following select deconvolved bands: amide (Am) III $\beta$-sheet/random coil ( $\sim 1248 \mathrm{~cm}^{-1}$; contains minor contributions from cytosine), Am III $\alpha$-helix $\left(\sim 1266 \mathrm{~cm}^{-1}\right)$, Am I $\alpha$-helix $\left(\sim 1656 \mathrm{~cm}^{-1}\right)$, and Am I disordered $\left(\sim 1685 \mathrm{~cm}^{-1}\right.$, mainly $\beta$-turns $){ }^{23,33,34}$ Other bands shown in Fig. 1 include the phenylalanine (Phe) and mixed protein/lipid $\mathrm{CH}_{2}$ deformation bands at $\sim 1002$ and $\sim 1447 \mathrm{~cm}^{-1}$, respectively.

\subsection{Spectral Assignment of the Green Fluorescence Protein Chromophore}

A closer inspection of the mean spectra shown in Figs. 1(f)-1(h) revealed an additional band at $\sim 1552 \mathrm{~cm}^{-1}$ that appeared exclusively for the gene-silenced UC13-Scr, UC13-A, and UC13-C cell lines. Initially, the band was attributed to Typ because its position approximately coincided with the 1546- and $1552-\mathrm{cm}^{-1}$ Typ band assignments reported for bladder and lung tissue cancers, respectively. ${ }^{35,36}$ However, the absence of a proportionally intense Typ band around $757 \mathrm{~cm}^{-1}$ in the spectra of gene-silenced UC13 cell lines, which is a characteristic of pure Typ and Typ-rich proteins, ${ }^{37,38}$ suggested a different band assignment. A GFP band assignment was then proposed because only the gene-silenced UC13 cell lines fluoresced green when exposed to UV-light. Indeed, published Raman spectra of the anionic form of GFP and its structural variants contained a prominent band between 1537 and $1556 \mathrm{~cm}^{-1}$ that was assigned to the $-\mathrm{C}=\mathrm{C}-\mathrm{C}=\mathrm{N}-$ portion of the imidazolinone ring. ${ }^{6,7}$ This assignment was further corroborated by our fluorescence imaging and Raman ratiometric studies. As shown by the fluorescence images of UC13 cell lines in Figs. 3(a)-3(d), the fluorescence intensity of GFP (with respect to UC13Con cells) appeared to increase in the following order: UC13-A > UC13-C > UC13-Scr. A similar order was found when the ratio intensities of the GFP band at $\sim 1552 \mathrm{~cm}^{-1}$ to the protein Am I $\alpha$-helix band at $\sim 1656 \mathrm{~cm}^{-1}$ (GFP/Am I $\alpha$-helix ratio) were compared [Fig. 4(a)]. This is the first Raman spectroscopic study in which the GFP chromophore has been correctly identified in cancer cells transduced with the GFP reporter gene.

The near-absence of GFP expression from the fluorescent images obtained for the gene-silenced T24 cell lines (T24Scr, T24-A, and T24-C) in Figs. 3(f)-3(h) suggests that either GFP transduction was low or that the cyclization of the GFP tripeptide to the fluorescent chromophore was inefficient. By comparing the GFP/Am I $\alpha$-helix ratios, the T24-C cell line exhibited some measureable GFP expression but was not statistically significant [Fig. 4(a)]. This is not entirely unexpected. Additionally, transduced cells with low GFP expression are sometimes preferred because they exhibit morphological and biochemical traits that are consistent with their parental cell line under study. ${ }^{8}$ Moreover, panel cell line studies have shown that UMUC-13 cells can be effectively transduced with the GFP reporter gene with negative toxicity as assessed by light microscopy at 48 h. ${ }^{11}$ Regardless of whether the gene-silenced UC13 cell lines exhibited low- or high-GFP expression, the gene-silenced cells shown in Fig. 3 appeared to be healthy and exhibited cell morphologies that were similar to their parental counterparts. (a)

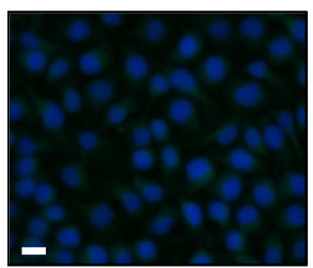

(b)
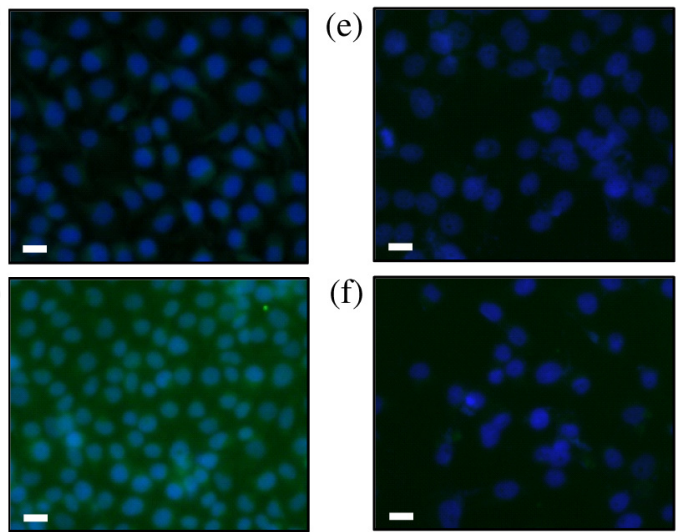

(f)

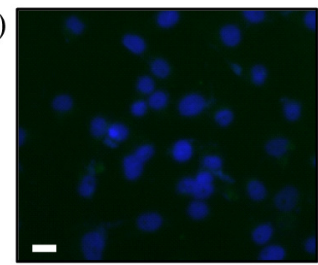

(c)

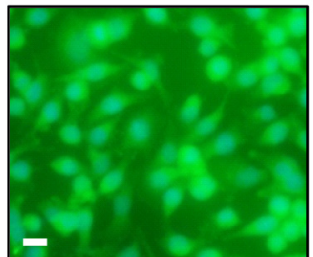

(g)

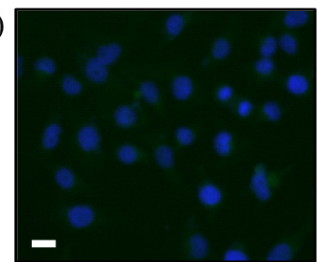

(d)

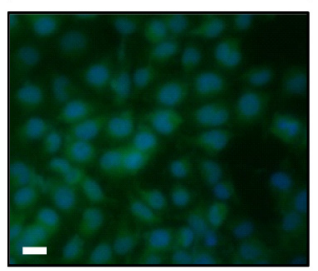

(h)

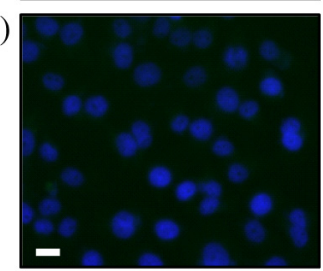

Fig. 3 Representative merged nuclear stained (blue, due to DAPI) and GFP (green) fluorescence images obtained for (a) UC13-Con, (b) UC13-Scr, (c) UC13-A, (d) UC13-C, (e) T24-Con, (f) T24-Scr, (g) T24-A, and (h) T24-C cell lines. The UC13-A cell line (c) exhibited the highest GFP fluorescence, followed by the UC13-C (d) and UC13Scr (b) cell lines. GFP fluorescence was negligible for the corresponding gene-silenced T24 cell lines (f-h) and parental UC13 (a) and T24 (e) cell lines. Scale bar: $20 \mu \mathrm{m}$.

\subsection{Spectral Biochemical and Protein Structural Analyses}

To evaluate biochemical and protein structural differences between parental and gene-silenced cell lines, several ratiometric Raman parameters as shown in Fig. 4 were examined. Initially, nucleic acid/Typ ratios were examined because the parameter had previously been used to distinguish between normal human skin cells and tumorigenic keratinocytes. ${ }^{19}$ Changes in nucleic acid/Typ ratios can provide an insight into the differentiation status of cells, either living or dried. ${ }^{20,27}$ The nucleic acid/Typ ratio was significantly increased for UC13-Scr cells but decreased for T24-A cells relative to their parental controls [Fig. 4(b)]. In addition, nucleic acid/Typ ratios were increased for UC13-Scr and UC13-C cells relative to T24-Scr and T24-C cells. This suggests that the parental UC13 cell line was more sensitive to shRNA-mediated gene silencing compared to the parental T24 cell line. The high nucleic acid and low protein content exhibited by UC13-Scr and UC13-C cell lines suggest a more undifferentiated cell phenotype, whereas T24-Scr and T24-C cells with their lower nucleic acid but higher protein content suggest a more differentiated cell phenotype. ${ }^{20,39}$ Nevertheless, high nuclear DNA content was consistent with other bladder cancer cells and tissues studied by Raman spectroscopy. ${ }^{40}$ 

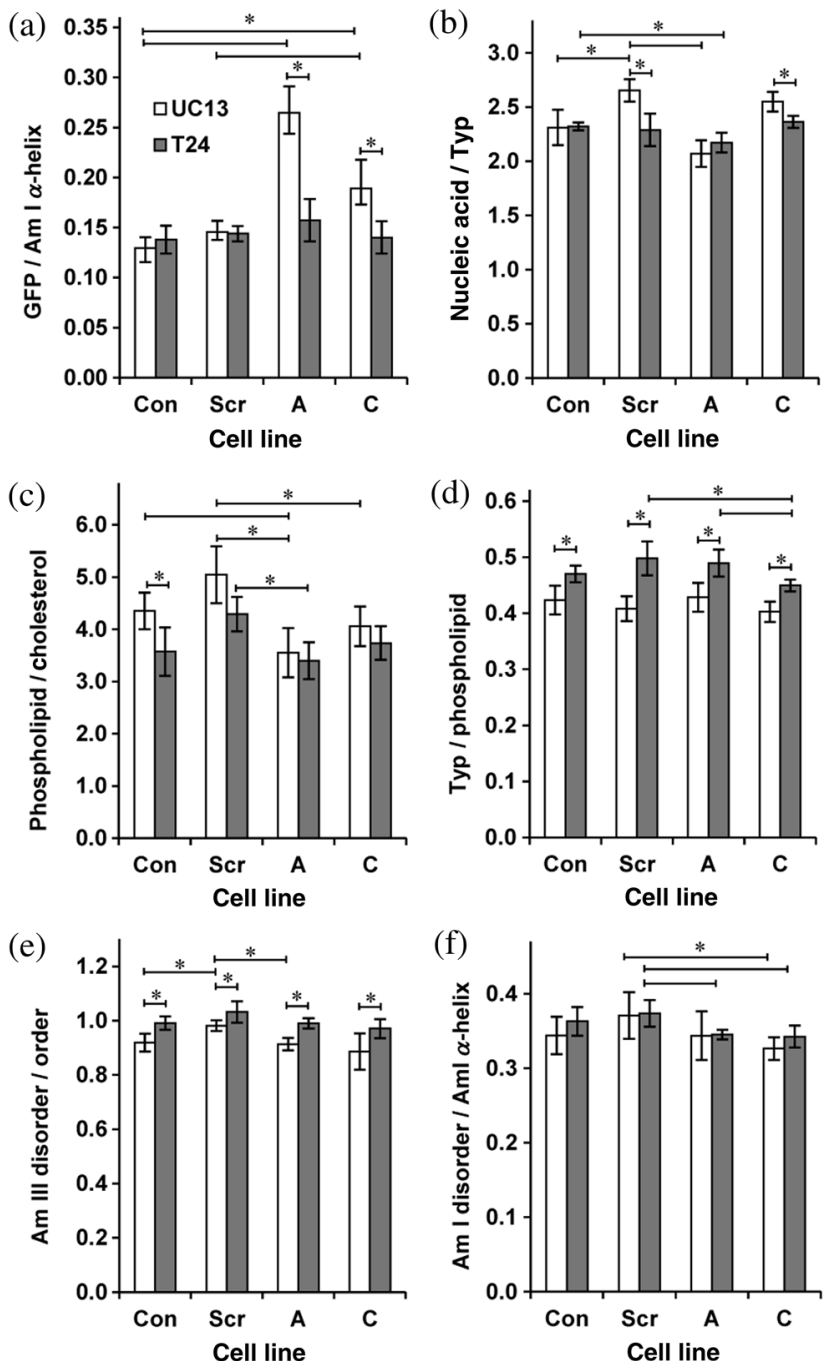

Fig. 4 Relative distributions of biochemical and protein secondary structural components obtained by spectral fitting of parental (Con), scrambled (Scr), and gene-silenced (A or C) T24 and UC13 (A or C) cell lines. Plot error bars are based on SDs $(n=5$ per cell line). ${ }^{*} p$ values are defined at $<0.05$.

Phospholipid metabolism, particularly phosphocholine and total choline, is known to be elevated in breast, brain, and prostate cancers because of the overexpression of choline kinase- $\alpha$ $(\mathrm{ChoK} \alpha)$, the enzyme involved in their biosynthesis. ${ }^{41}$ Elevated $\mathrm{ChoK} \alpha$ and phosphocholine levels are also associated with a more aggressive phenotype for certain bladder carcinomas. ${ }^{42}$ As shown in Fig. 4(c), significant increases in phospholipid/cholesterol ratios were observed between parental and between some gene-silenced UC13 and T24 cell lines. This parameter was chosen because changes in phospholipid/cholesterol ratios had previously been stratified against known glioma tumor grades. ${ }^{21}$ In addition, a recent Raman tissue imaging study has shown that prostate cancer progression could be stratified according to accumulated cholesterol esters, which were elevated in high-grade and metastatic prostate cancers. ${ }^{43}$ As shown in Fig. 4(c), phospholipid/cholesterol ratios were slightly reduced across all T24 cell lines but not across UC13 cell lines.

Although comparative literature lipogenic profiles on both UC13 and T24 cell lines are scarce, there is one study in which the de novo lipid synthesis by T24 cells were compared against other cancer cell lines. ${ }^{42}$ Compared to prostate (PC3M), lung (HO62), and hepatocellular (HepG2) carcinoma cells, T24 cells displayed a low lipogenic phenotype under standard cell culture conditions. ${ }^{42}$ This could account for the observation that T24 cell lines demonstrated a trend of lower phospholipid/cholesterol ratios compared to UC13 cells in Fig. 4(c), including why all four T24 cell lines consistently exhibited higher Typ/phospholipid ratios compared to UC13 cell lines in Fig. 4(c). Unfortunately, due to spectral overlap between the $\mathrm{C}-\mathrm{N}$ stretching mode of the polar choline group of phosphocholine and free choline components, ${ }^{44}$ it was not possible to measure their relative intensity ratios. Although the spectral signature of free choline in cancer cells has yet to be identified directly, a recent isotope-labeled Raman imaging study showed that living cancer cells contained higher amounts of deuterated choline metabolites inside the nucleus compared to noncancer cells. $^{45}$

The surprisingly high nucleic acid/Typ and phospholipid/ cholesterol ratios exhibited by UC13-Scr cells, the cell phenotype with the lowest GFP/Am I $\alpha$-helix ratio, implied that shRNA gene silencing had a more profound effect on cancer cell biochemistry than GFP expression. To determine whether gene silencing could also influence protein secondary structure, additional ratiometric parameters derived from deconvolved Am I and Am III subbands were examined. As shown in Fig. 4(e), the Am III disorder/order ratios were significantly higher for UC13-Scr cells than UC13-Con and UC13-A cells. A similar trend was found when the Am I disorder/Am I $\alpha$-helix ratios were examined, but the results were not statistically significant [Fig. 4(f)]. However, Am I disorder/Am I $\alpha$-helix ratios were found to be significantly higher for UC13-Src and T24-Scr compared to UC13-C and T24-C cells, respectively. The finding that Raman spectroscopy can detect changes in bladder cancer cell protein secondary structure is supported by other spectroscopic studies involving colorectal cancer tissues ${ }^{46}$ and colon cancer cells. ${ }^{23}$ Moreover, some cancer-associated proteins are intrinsically more disordered and are known to be associated with increased cancer risk. ${ }^{47-49}$

\section{Conclusions}

In this study, we proposed that Raman microscopy could not only be used to distinguish between parental and mutated T24 and UC13 bladder cancer cell lines but also to determine whether gene-silenced cells expressing GFP could perturb their biochemical and protein structural properties. First, Raman spectra of the three gene silenced UC13 cell lines contained an additional band at $\sim 1552 \mathrm{~cm}^{-1}$ that appeared to vary in intensity, but was consistent for a given cell phenotype. Fluorescence imaging and Raman ratiometric studies showed that this band was attributed to GFP and not Typ as initially proposed. This is the first Raman spectroscopic study in which the spectral signature of the GFP reporter gene has been identified in transduced cancer cells. Second, we successfully demonstrated that shRNA gene silencing and cancer cell type had a more profound effect on nucleic acid and lipogenic metabolism than did GFP expression. This finding was consistent with results obtained from the spectral analysis of cellular protein secondary structure. Indeed, the relative amounts of protein order and protein disorder appeared to be influenced by the site of shRNA gene silencing, whereas nonspecific (scrambled) shRNA gene silencing gave rise to cell phenotypes with higher protein disorder. Although cancer-associated proteins are intrinsically 
more disordered, we speculate that nonspecific gene silencing may have enhanced the expression and/or lengthened the time disordered proteins spent at specific phases of the cell cycle. Future flow cytometry and in vivo Raman microscopy studies will be required to clarify the interplay between cancer cell cycle and protein secondary structure.

\section{Acknowledgments}

GSM and MDM acknowledge funding support under Grant No. R01-AR054496 from the National Institute of Arthritis and Musculoskeletal and Skin Diseases. GSM thanks Alexander Zaslavsky from the Department of Urology, University of Michigan, for providing guidance with fluorescence staining and imaging. ETK and ALH acknowledge funding support from the National Institutes of Health (NIH) under Grant No. P01CA093900. GSM, ALH, ETK, and MDM declare that they have no conflicts of interest.

\section{References}

1. D. M. Chudakov et al., "Fluorescent proteins and their applications in imaging living cells and tissues," Physiol. Rev. 90(3), 1103-1163 (2010).

2. R. M. Hoffman, "Application of GFP imaging in cancer," Lab. Invest. 95(4), 432-452 (2015).

3. G. N. Phillips, "Structure and dynamics of green fluorescent protein," Curr. Opin. Struct. Biol. 7(6), 821-827 (1997).

4. M. Zimmer, "Green fluorescent protein (GFP): applications, structure, and related photophysical behavior," Chem. Rev. 102(3), 759-781 (2002).

5. S. E. Jackson, T. D Craggs, and J. R. Huang, "Understanding the folding of GFP using biophysical techniques," Expert Rev. Proteomics 3(5), 545-559 (2006).

6. A. F. Bell et al., "Light-driven decarboxylation of wild-type green fluorescent protein," J. Am. Chem. Soc. 125(23), 6919-6926 (2003).

7. X. He, A. F. Bell, and P. J. Tonge, "Isotopic labeling and normal-mode analysis of a model green fluorescent protein chromophore," J. Phys. Chem. B 106(23), 6056-6066 (2002).

8. J. M. Tavare, L. M. Fletcher, and G. I. Welsh, "Review-using green fluorescent protein to study intracellular signalling," J. Endocrinol. 170(2), 297-306 (2001).

9. J. V. F. Coumans et al., "Green fluorescent protein expression triggers proteome changes in breast cancer cells," Exp. Cell Res. 320(1), 33-45 (2014).

10. J. Bubenik et al., "Established cell line of urinary-bladder carcinoma (T-24) containing tumor-specific antigen," Int. J. Cancer 11(3), 765-773 (1973).

11. A. Sabichi et al., "Characterization of a panel of cell lines derived from urothelial neoplasms: genetic alterations, growth in vivo and the relationship of adenoviral mediated gene transfer to coxsackie adenovirus receptor expression," J. Urol. 175(3), 1133-1137 (2006).

12. M. M. Mariani et al., "Impact of fixation on in vitro cell culture lines monitored with Raman spectroscopy," Analyst 134(6), 1154-1161 (2009).

13. U. Neugebauer et al., "Identification and differentiation of single cells from peripheral blood by Raman spectroscopic imaging," J. Biophotonics 3(8-9), 579-587 (2010).

14. P. A. Felice et al., "Raman spectroscopy delineates radiation-induced injury and partial rescue by amifostine in bone: a murine mandibular model," J. Bone Min. Metab. 33(3), 279-284 (2015).

15. N. J. Crane et al., "Raman spectroscopic evidence for octacalcium phosphate and other transient mineral species deposited during intramembranous mineralization," Bone 39(3), 434-442 (2006).

16. F. W. L. Esmonde-White, K. A. Esmonde-White, and M. D. Morris, "Minor distortions with major consequences: correcting distortions in imaging spectrographs," Appl. Spectrosc. 65(1), 85-98 (2011).

17. S. Banerjee and D. Y. Li, "Interpreting multicomponent infraredspectra by derivative minimization," Appl. Spectrosc. 45(6), 1047-1049 (1991).
18. I. Notingher et al., "Spectroscopic study of human lung epithelial cells (A549) in culture: living cells versus dead cells," Biopolymers 72(4), 230-240 (2003).

19. P. Piredda et al., "Subcellular Raman microspectroscopy imaging of nucleic acids and tryptophan for distinction of normal human skin cells and tumorigenic keratinocytes," Anal. Chem. 87(13), 6778-6785 (2015).

20. H. G. Schulze et al., "Assessing differentiation status of human embryonic stem cells noninvasively using Raman microspectroscopy," Anal. Chem. 82(12), 5020-5027 (2010).

21. M. Köhler et al., "Characterization of lipid extracts from brain tissue and tumors using Raman spectroscopy and mass spectrometry," Anal. Bioanal. Chem. 393(5), 1513-1520 (2009).

22. G. S. Mandair and M. D. Morris, "Contributions of Raman spectroscopy to the understanding of bone strength," Bone Key Rep. 4, 620 (2015).

23. S. Akyuz et al., "Raman micro-spectroscopic analysis of cultured HCT116 colon cancer cells in the presence of roscovitine," Spectrosc. Acta Pt. A-Mol. Biomol. Spectrosc. 78(5), 1540-1547 (2011).

24. H. Bai et al., "Detecting viability transitions of umbilical cord mesenchymal stem cells by Raman micro-spectroscopy," Laser Phys. Lett. 8(1), 78-84 (2011).

25. L. T. Kerr, H. J. Byrne, and B. M. Hennelly, "Optimal choice of sample substrate and laser wavelength for Raman spectroscopic analysis of biological specimen," Anal. Methods 7(12), 5041-5052 (2015).

26. L. Mikoliunaite et al., "The substrate matters in the Raman spectroscopy analysis of cells," Sci. Rep. 5, 13150 (2015).

27. S. O. Konorov et al., "Raman microspectroscopic evidence that dryfixing preserves the temporal pattern of non-specific differentiation in live human embryonic stem cells," J. Raman Spectrosc. 42(4), 576-579 (2011).

28. A. I. Mazur et al., "Evaluating different fixation protocols for spectral cytopathology. Part 2: cultured cells," Anal. Chem. 84(19), 8265-8271 (2012).

29. S. Dochow et al., "Tumour cell identification by means of Raman spectroscopy in combination with optical traps and microfluidic environments," Lab Chip 11(8), 1484-1490 (2011).

30. A. N. Kuzmin, A. Pliss, and P. N. Prasad, "Changes in biomolecular profile in a single nucleolus during cell fixation," Anal. Chem. 86(21), 10909-10916 (2014).

31. V. Ranc et al., "Discrimination of circulating tumor cells of breast cancer and colorectal cancer from normal human mononuclear cells using Raman spectroscopy," Analyst 138(20), 5983-5988 (2013).

32. C. Krafft et al., "Near infrared Raman spectra of human brain lipids," Spectrosc. Acta Pt. A-Mol. Biomol. Spectrosc. 61(7), 1529-1535 (2005).

33. Z. Movasaghi, S. Rehman, and I. U. Rehman, "Raman spectroscopy of biological tissues," Appl. Spectrosc. Rev. 42(5), 493-541 (2007).

34. S. U. Sane, S. M. Cramer, and T. M. Przybycien, "A holistic approach to protein secondary structure characterization using amide I band Raman spectroscopy," Anal. Biochem. 269(2), 255-272 (1999).

35. W. L. Zha et al., "HPLC assisted Raman spectroscopic studies on bladder cancer," Laser Phys. Lett. 12(4), 045701 (2015).

36. Z. W. Huang et al., "Near-infrared Raman spectroscopy for optical diagnosis of lung cancer," Int. J. Cancer 107(6), 1047-1052 (2003).

37. B. Hernandez et al., "Vibrational analysis of amino acids and short peptides in hydrated media. VIII. Amino acids with aromatic side chains: Lphenylalanine, L-tyrosine, and L-tryptophan," J. Phys. Chem. B 114(46), 15319-15330 (2010).

38. B. Sjoberg et al., "An experimental and theoretical study of the amino acid side chain Raman bands in proteins," Spectrosc. Acta Pt. A-Mol. Biomol. Spectr. 128, 300-311 (2014).

39. S. Barkur et al., "Probing differentiation in cancer cell lines by singlecell micro-Raman spectroscopy," J. Biomed. Opt. 20(8), 085001 (2015).

40. E. Canetta et al., "Discrimination of bladder cancer cells from normal urothelial cells with high specificity and sensitivity: combined application of atomic force microscopy and modulated Raman spectroscopy," Acta Biomater. 10(5), 2043-2055 (2014).

41. K. Glunde, Z. M. Bhujwalla, and S. M. Ronen, "Choline metabolism in malignant transformation," Nat. Rev. Cancer 11(12), 835-848 (2011).

42. E. Hernando et al., "A critical role for choline kinase-alpha in the aggressiveness of bladder carcinomas," Oncogene 28(26), 2425-2435 (2009). 
43. S. H. Yue et al., "Cholesteryl ester accumulation induced by PTEN loss and PI3K/AKT activation underlies human prostate cancer aggressiveness," Cell Metab. 19(3), 393-406 (2014).

44. H. Akutsu, "Direct determination by Raman-scattering of the conformation of the choline group in phospholipid-bilayers," Biochemistry 20(26), 7359-7366 (1981).

45. F. H. Hu et al., "Live-cell vibrational imaging of choline metabolites by stimulated Raman scattering coupled with isotope-based metabolic labeling," Analyst 139(10), 2312-2317 (2014).

46. P. C. Lopes et al., "Discriminating adenocarcinoma from normal colonic mucosa through deconvolution of Raman spectra," J. Biomed. Opt. 16(12), 127001 (2011).
47. F. X. Theillet et al., "Physicochemical properties of cells and their effects on intrinsically disordered proteins (IDPs)," Chem. Rev. 114(13), 6661-6714 (2014).

48. A. L. Fink, "Natively unfolded proteins," Curr. Opin. Struct. Biol. 15(1), 35-41 (2005).

49. V. N. Uversky, C. J. Oldfield, and A. K. Dunker, "Intrinsically disordered proteins in human diseases: introducing the D(2) concept," Annu. Rev. Biophys. 37, 215-246 (2008).

Biographies for the authors are not available. 